\title{
e-Phaïstos
}

e-Phaïstos

Revue d'histoire des techniques / Journal of the history

of technology

V-2 | 2016

Histoire des techniques en Afrique de l'Ouest

\section{Édifier et équiper les bases de l'armée japonaise : Transferts de technologie, France-Japon 1868-1930}

\section{Michiko Maejima}

\section{(2) OpenEdition}

Journals

Édition électronique

URL : http://journals.openedition.org/ephaistos/6053

DOI : 10.4000/ephaistos.6053

ISSN : 2552-0741

Éditeur

IHMC - Institut d'histoire moderne et contemporaine (UMR 8066)

Édition imprimée

Date de publication : 15 juin 2016

Pagination : 116-120

ISSN : 2262-7340

Référence électronique

Michiko Maejima, «Édifier et équiper les bases de l'armée japonaise : Transferts de technologie,

France-Japon 1868-1930 », e-Phaïstos [En ligne], V-2 | 2016, mis en ligne le 15 mars 2020, consulté le 15 septembre 2020. URL : http://journals.openedition.org/ephaistos/6053 


\title{
Michiko Maejima
}

\section{Édifier et équiper les bases de l'armée japonaise Transferts de technologie France-Japon 1868-1930}

\author{
Thèse de doctorat d'histoire \\ Conservatoire national des arts et métiers
}

Histoire, techniques, territoire, patrimoine (HTTP) - Histoire des technosciences en société (HT2S)

Soutenue le 26 mai 2014

Un volume (470 p.) - un volume d'annexes (230 p.)

Directeurs de thèse :

André GUILLERME, Professeur au CNAM

Jury:

Philippe BONNIN, Directeur de recherches CNRS, UMR LaVue (rapporteur)

Karen BOWIE, Professeure, École Nationale Supérieure d'Architecture de Paris La Villette (présidente du jury)

Christian BRUN, Directeur de recherches à l'École de l'Air, Salon-de-Provence

Riichi MIYAKE, Professeur à l'Université des Femmes de Fuji, Sapporo, Japon (rapporteur)
Madame la présidente,

Mesdames et Messieurs les Membres du Jury,

Cette recherche, je l'ai conçue au cours de mes études supérieures au Japon puis en France alors que j'étudiais la civilisation française. Le patrimoine architectural civil est largement analysé et protégé au Japon, mais le patrimoine militaire reste sousévalué et très peu apprécié de l'opinion publique japonaise pacifiste qui trouve cet héritage militaire encombrant. Le Ministre de la Défense pressé de faire l'inventaire de ces biens, a fait appel à des experts, dont le professeur Riichi Miyake qui m'encouragé à participer à cette reconnaissance militaire originale.

Comme beaucoup de francophones japonais, j'ai trouvé qu'il y avait de la fin du $19^{\mathrm{e}}$ siècle jusqu'après la première guerre mondiale, des relations fortes entre la France et le Japon. Pourtant, aujourd'hui, au Japon, l'opinion publique estime que les EtatsUnis ont été le modèle à suivre avec l'Allemagne et que l'armée japonaise moderne a été créée selon le modèle prussien. Effectivement, après la défaite cuisante de la France en 1870-71, la Prusse y envoie des officiers instructeurs pour éduquer les officiers et en faire des alliés, mais alors on comprend mal pourquoi le Japon s'allie à la France et à la Grande Bretagne en 1915, contre l'Allemagne. Mes recherches contestent cette origine germanophile de 
l'armée nippone et apportent les preuves archivistiques qu'il y avait des relations militaires très intenses entre la France et le Japon, notamment entre 1868 et 1930. L'amnésie semble avoir été surtout entretenue à partir du moment où le Japon est devenu un Etat totalitaire, et a noué une alliance obligée avec l'Allemagne, c'est-à-dire le début des années 1930. Mais ceci est une autre histoire, une autre thèse à déposer.

La première mission militaire française est envoyée au Japon en 1868, juste avant la Révolution Meiji, An o de la modernisation. Le pays est alors sous pression : les puissances américaine et européennes exigent l'ouverture des ports de commerce. Pour la première fois le gouvernement Edo, réduit à merci, conçoit d'établir une armée qualifiée de «moderne » c'est-à-dire capable de s'opposer à ces puissances.

Cependant, la mission tourne court avec la révolution : quelques membres de cette mission démissionnent pour combattre du côté de l'ancien gouvernement Edo, et se retranchent dans la forteresse de Goryokaku, à Hakodaté. Quel impact a ce fait d'armes auprès des élites française et japonaise ? Malgré la fidélité des officiers français au gouvernement Edo, le nouveau, Meiji, adopte aussi le modèle français de casernement : des bâtiments pour éduquer, controler, et surveiller le guerrier nouveau : un travail pour le Génie ? Quel est le processus d'élaboration du plan ? Y-a-t-il un modèle de caserne spécifiquement français ? Celui du colonel Belmas ? Un modèle allemand ? La clé est le Capitaine Jourdan (1840-1898), membre de la première mission puis de la deuxième durant laquelle il élabore les premières architectures militaires du Soleil levant.

La construction des casernes est tout à fait originale et originelle, incomparable avec l'architecture monumentale japonaise. En 1871, cette nouvelle architecture est prise en charge par le département de la Construction du ministère de la Guerre. Jourdan est au Japon l'année suivante. Un an passe et le service devient le « $4^{\mathrm{e}}$ département » composée d'ingénieurs, de conducteurs et de gardemine $^{1}$ chargés d'adapter localement la construction et d'inspecter les travaux. Le capitaine du Génie y joue un rôle de conseil et participe à la création de la « Direction » qui devient autonome en 1879 sous le titre «Département du Génie ». Parmi les collaborateurs de Jourdan: Shigeto Nakamura (1840-1884), officier du Génie, le Viollet-le-Duc japonais qui a sauvagardé plusieurs châteaux alors que l'idée du gouvernement est de substituer des casernes "modernes aux forteresses.

Le Japon adopte le système militaire français et place ses casernes dans chaque Tchintaï - Division - , du $1^{\text {er }}$ au $14^{\mathrm{e}}$ Régiment d'Infanterie. Dans l'Infanterie, quatre des plus anciennes casernes, construites en 1873 et 1874 à Sendai², Aomori3, Nagoya 4 et Shibata 5 existent toujours. Elles partagent le même type de charpente que la filature de soie de Tomioka, bâtie entre 1870 et 1872 , inscrite sur la liste du patrimoine mondial de l'Unesco en juin 2014, et que la résidence du sousdirecteur de la forge de Yokosuka dressée vers 1871 : elles ne sont pas de tradition japonaise. Les quatre casernes sont édifiées sur le modèle dessiné par Jourdan. La filature et la Résidence sont dessinées par un français, civil, Edmond Bastien (1839-1888) qui est au Japon en même temps que Jourdan, partageant amis, voyages, et randonnées : il y a échanges d'informations et de savoir-faire.

Selon J. Edwards ${ }^{6}$, aux Etats-Unis, ce type de charpente est appelé "vernacular french truss » dans l'ancienne colonie française. Elle est largement diffusée dans les années 1920 par le Colonel Espitallier.

Une photo prise par le Lieutenant Louis Kreitmann (1851-1914), membre de la deuxième mission, futur général, montre les casernes du Génie construites par Jourdan à Tokyo. Quatre casernes identiques autour d'une cour ont des points communs avec d'autres casernes, à un rapport d'échelle près.

Pour les casernes, les bases militaires, une cours d’intérieur carrée entourée par des bâtiments, sont 
pareilles aussi. Le modèle de casernement est publié dans le Mémorial des Officiers du Génie que lisent les ingénieurs militaires français, publié en 1848 par le Capitaine Belmas. Le Manuel de construction française traduit et publié au Japon en 1870 se réfère à la version hollandaise parce qu'alors les Pays-Bas étaient le seul pays européen autorisé à commercer. Mais le manuel présente bien l'article de Belmas ainsi que ses plans, dessinés à nouveau, un peu simplifiés, par la main japonaise. J'ai pu assister au démantèlement pièce par pièce de la caserne de Shibata, et justifier mes hypothèses.

En 1870, dix jeunes japonais destinés à servir dans l'armée viennent en France étudier. Sanshichiro Ishimaru (1850-1895 ?) et Iwao Oguni (1856-1901) qui y séjourne dix ans, occupent à leur retour des rôles stratégiques. Ce dernier enseigne à l'École militaire et promeut la construction de batteries côtières.

Alors que ces élèves-officiers voguent vers la France, une première mission française part au Japon. Elle débarque en 1872. De fait, les communications sont intenses et sincères. Même après le départ des Français, les Japonais entretiennent précieusement ces bâtiments, mémoires d'Outre-pacifique, ainsi que la conception : le plan standard est reproductible.

Le lieutenant-colonel Meunier propose de défendre la côte à l'aide de batteries. La conception date de 1880 : à nouveau une mission japonaise part pour la France. Oguni n'est pas encore rentré ; il fait partie des quelques officiers qui assurent ainsi une sorte d'alliance franco-japonaise.

Pour l'artillerie, les officiers comme le capitaine Jules Brunet (1838-1911) y sont aussi dès la première mission : le Japon veut doter ses côtes de canons. Pour la France, une aubaine politique et commercial : ainsi Schneider exporte ses canons à partir des années 1890. Dix ans plus tard, des artilleurs japonais viennent en France pour étudier. Le prince Nashimoto visite Schneider au Creusot en 1909.
Dans la Cavalerie, dont la fonction est noble, voire impériale, Yoshifuru Akiyama (1859-1930), le père de la cavalerie japonaise travaille sous la direction de l'Allemand Klemens Wilhelm Jacob Meckel (1842-1906). En 1887, Akiyama sorti de l'École, rejoint Sadakoto Hisamatsu (1867-1943), l'ancien chef de clan Matsuyama, qui séjourne en France depuis 1883 pour apprendre la guerre. Akiyama suit l'École de Saint-Cyr trois ans durant. L'armée japonaise adopte le modèle allemand, mais Akiyama considère la cavalerie française supérieure; l'allemande étant trop formelle et dépourvue de stratégie, alors que la cavalerie française est jugée plus tactique, tout terrain, ce qui est fondamental pour les conquêtes coloniales en Chine ou dans les îles Sakarine. Lors d'une conférence avec Aritomo Yamagata, ministre de l'Armée, Akiyama lui démontre tout l'intérêt qu'il y a à continuer la structure française de cavalerie. Le célèbre auteur de romans historiques, Ryorato Shiba, en a bien fait la chronique. Hisamatsu, chef du clan Matsuyama, séjourne huit ans en France. À son retour, il remplace le consul français au Japon; puis revient en France comme attaché militaire et finit lieutenant au Japon.

Des manèges sont dessinés par Jourdan, selon les photos et des notes prises par Louis Kreitmann. Par la suite, les ingénieurs japonais ont développé ces prototypes, sans pilier central, notamment au nord du Japon.

Et cette coopération technologique continue dans la conception et la construction d'hangars pour avion. Une troisième mission forte de soixante hommes vient initier l'armée de l'Air en 1918. Pour les mess d'officiers d'aviation aussi, bien que l'on raconte que ce sont des ingénieurs japonais qui ont construits ces bâtiments très européens et non pas les officiers français. Or l'un des responsables, Yasushi Tamura (1878-1942) est venu en France pour visiter les bases et étudier les architectures selon le document des archives du Ministère des Affaires Etrangères. 
Les mess d'officiers d'aviation d'allure occidentale conçus par les ingénieurs Tamura et Mosuke Sato (1884-1921), ne sont pas une pâle copie des mess français : même si Tamura s'est rendu en France et en Europe, il y est resté peu de temps. En réalité, les ingénieurs nippons sont tout à fait mûrs pour élaborer des mess qui rendent compte d'une hégémonie architecturale, une originalité toute impériale. Oguni, officier du Génie, envoyé en France pour étudier pendant neuf ans, a parfaitement assuré le relai.

Quand la troisième mission d'aviation va au Japon, les ministres des Armées étaient tour à tour Shinroku Ishimoto (1854-1912) et Yusaku Uéhara (1856-1933), le père du Génie japonais, et Yukihiko Kusunosé (1858-1927), trois officiers qui avaient étudié l'artillerie en France trente-cinq ans auparavant... Cette troisième mission était voulue et parfaitement organisée. Après vous avoir présenté les principaux résultats de ma thèse, j'aimerai revenir sur ma façon de travailler.

Les documents papiers sont rares, tout particulièrement au Japon. La plupart ont quasiment été détruits par l'armée japonaise à la fin de la deuxième guerre mondiale, ou par les incendies ou par les bombardements américains. À cela s'ajoute que la conservation des archives n'a pas la même valeur qu'en France (on conserve beaucoup moins). De fait au Japon, on a plus une attitude d'archéologue qu'une posture d'historien : on travaille sur des témoignages et des vestiges, et moins avec des textes et de l'iconographie. J'ai participé sous la direction du professeur Miyaké, à l'inventaire d'une des plus anciennes casernes à Shibata, remontée pièce par pièce pour être le premier musée militaire, inauguré en 2014. J'en ai fait une thèse japonaise soutenue à Tokyo en février 2012.

Shibata est bien un modèle d'architecture militaire. Cela étant, chaque caserne possède une typicité liée au climat, au sol, aux temporalités. Au début de cette militarisation du territoire, il semble qu'on veuille n'utiliser que des matériaux neufs pour répondre au dimensionnement imposé mais, de fait, coûteux et rare. On récupère les bois, pierres, et tout ce qui pouvait être utile sur le château féodal proche. On recycle les matériaux, on donne à nouveau vie et force aux nouveaux bâtiments.

Les finitions de plâtre intérieures et extérieures ont un effet esthétique certain qu'on retrouve encore dans les façades des châteaux féodaux toujours raffinés selon l'idéologie chevaleresque. En ce sens, si la caserne se réfère structurellement à l'Occident, dans la forme elle reste japonaise. Shigeto Nakamura défend ces caractères traditionnels. Même si le château de Shibata est petit, comparé à ceux de Himeji et Nagoya, en conservant un maximum de bâti, on a créé avec cette caserne Shirakabé - en français, «Mur blanche »- un ensemble certes anachronique mais harmonieux.

Comme beaucoup de chercheurs japonais, j'ai eu quelques difficultés à travailler les textes de la fin d'Edo qui sont très différemment calligraphiés. Même à l'époque Meiji, les mots étrangers sont bizarrement écrits. Ainsi, Chéry se prononce "chélie", Mougin se pronounce "mouzén" ou "moujïan". Et "chélie" ne donne pas un nom d'auteur en français, ni un nom d'officier quand j'ai parcouru les fichiers militaires du Service Historique de la Défense à Vincennes à la recherche de l'auteur du Traité de l'art militaire contenant l'architecture des casernes.

A contrario, j'ai eu de la chance de trouver de magnifiques archives, notamment les grandes collections de photos et de documents ramenés et déposés au Collège de France par Louis Kreitmann. Le fonds Schneider au Creusot m'a permis de prouver l'armement lourd du Japon autour de 1900. Pour l'aviation, le Musée du Bourget et le Service Historique de la Défense à Vincennes possèdent des documents de première importance quant à la naissance de l'aviation japonaise après la première guerre mondiale. Enfin le fonds 
Descharmes au musée de l'Empéri à Salon-deProvence révèle tout ce qui a trait à la vie quotidienne des officiers français au Japon. Les archives du Ministère des Affaires Étrangères à Paris sont aussi remarquables et montrent les liens solides entre la France et le Japon, notamment sur l'architecture militaire.

Si la France a autant marqué l'armée japonaise, c'est probablement parce qu'elle avait la première armée du monde à la fin du XIXe siècle. Les Prussiens qui prennent la suite de la formation de l'armée japonaise ne cherchent pas à imposer leur terminologie germanique par respect de l'armée française qu'ils ont longtemps admiré. C'est aussi parce que, pendant le Second Empire, la France est moins une puissance économique que la première nation aux frontières stables, avec une seule langue parlée, écrite et internationale (comme l'Empire romain); le plus puissant État doté d'une police, d'une armée, bref d'un appareil répressif qu'envient les empires russe, austro-hongrois, ottoman ; d'un appareil idéologique (éducation, religion) complémentaire. L’armée à la française permet au Japon impérial de devenir plus qu'une nation, un État.

Ces recherches conduisent à de nouvelles pistes. Côté japonais, au plan archéologique contemporain : une thèse relative à l'inventaire systématique des bâtiments militaires japonais pour le Ministère de la Défense permettrait d'évaluer le patrimoine. Côté français, il serait intéressant de connaître les constructions du Génie français en terre étrangère - Chine, Indochine, Afrique - et les comparer à celles des Britanniques en Inde, Chine, Nouvelle Zélande, ou des Hollandais en Indonésie. On sait notamment que tout ces États édifient des casernes à Pékin dans les années 1890 qui se présente comme une exposition universelle de casernes. Les articles de la Revue du Génie Militaire en font foi. La recherche et la conservation du patrimoine français dans le monde (colonial ou pas) mérite toute l'attention quant à la diffusion de la culture scientifique et technique.
La formation de l'armée de l'Air japonaise renvoie au rôle du Japon durant la première guerre mondiale : assistance ? observation ? espionnage ? Un colloque international sur la place du Japon dans la première guerre mondiale $\mathrm{y}$ apporterait beaucoup.

Je remercie les membres du jury qui ont manifesté leur intérêt pour mes travaux en acceptant d'être examinateurs et le public pour sa présence.

Mesdames et Messieurs, je vous remercie.

1 « Conditions du Génie », dans Horeï Zen-sho, 1873.

2 Ancienne caserne du 4e Régiment d'Infanterie

3 Ancienne caserne du 5e Régiment d'Infanterie

4 Ancienne caserne du 6e Régiment d'Infanterie

5 Ancienne caserne du 16e Régiment d'Infanterie

6 Jay D.Edwards "Upper Louisiana's French Vernacular Architecture in the Greater Atlantic World" Atlantic Studies. 8(4) : 2011, pp.411-445 